\title{
The Discrete Time Non-Persistent CSMA Protocol with Functions of Monitoring and Multichannel Mechanism Based on Binary Tree Conflict Resolution in WSN
}

\author{
S.J. Zhou, H.W. Ding, Y.F. Zhao, P. Li \\ School of Information \\ Yunnan University \\ Kunming, Yunnan, China
}

\begin{abstract}
In wireless sensor networks, the average cycle method is used to analyze the proposed protocol: the discrete time nonpersistent CSMA protocol with functions of monitoring and multichannel mechanism based on binary tree conflict resolution; accurate mathematical expressions of related indicators are got through rigorous mathematical derivation. By simulation, the correctness of the model is verified; by comparing to the system performance with the conventional non-persistent CSMA protocol, the former is more stable and controllable. On the basis of ensuring fairness in the allocation of resources, more network resources is assigned to the channel of higher priority, channel utilization improved greatly. With multichannel mechanism, system throughput has been significantly improved.
\end{abstract}

Keywords-wireless sensor network; conflict resolution; functions of monitoring; multichannel; non-persistent CSMA; throughput

\section{INTRODUCTION}

Wireless sensor networks (WSN) is a wired or wireless network consisting by sensors grouped in Ad hoc mode, with the purpose of collaboration and sensing, collection and processing the target information of geographical area covered by sensor networks, and passed to the observer [1]. The sensor network centralized the sensor technology, embedded computer technology and wireless communication technology; it senses cooperatively, monitors and collects the object perceived information under a variety of environment through the collaborative information processing, obtains the accurate information of the targets, and then transmits the information to the users who needs the information through the Ad hoc mode.

Non-persistent CSMA protocol with functions of monitoring is to add the monitoring information (ACK) to the end of each packet sent. Adding the monitoring information, the system can achieve monitoring the status of the network, fault alarm, identification intelligent, location, tracking network status, automatic measurement and control functions [2].

With the use of average cycle method, the proposed protocol improves the controllability of the system by increasing the ACK, taking up part of the network resources, making the throughput smaller compared with the conventional non-persistent CSMA protocol. Reduce the system collision rate by using the binary tree conflict resolution, multichannel technology increasing the system throughput, enhancing the controllability of the system, greatly improving the resource utilization of the whole system.

\section{THE MODEL}

The non-persistent CSMA protocol with functions of monitoring

Under the agreement control, there will be three random events: a packet is sent successfully(U events), the packet transmission is sent unsuccessfully(the collision appears, B events), the channel is idle (I events), these three events are forced into two events are: the channel is idle (I events) event and the channel is busy (BU event); the packet is sent successfully or unsuccessfully [3]. $T_{n}$ is a cycle period. $T P$ is the transmission period.

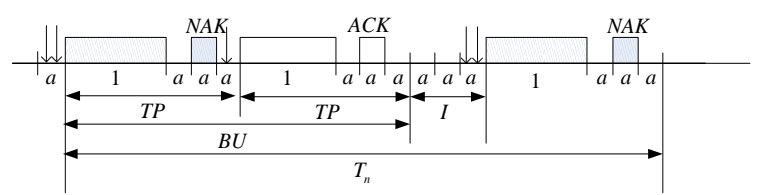

FIGURE I. THE MODEL OF NON-PERSISTENT CSMA PROTOCOL WITH FUNCTIONS OF MONITORING

\section{ANALYSIS OF THE MODEL}

The probability of the $\mathrm{n}$ arriving information packets in time ${ }^{t}$

In a cycle, the possibility of continuous $i$ idle I events and $j$ busy BU events is:

$$
P\left(N_{I}=i, N_{B U}=j\right)=\left(e^{-G a}\right)^{i-1}\left(1-e^{-G a}\right)\left(1-e^{-G(1+3 a)}\right)^{j-1} e^{-G(1+3 a)}
$$

The possibility of $E\left(N_{I}\right)$, the average number of $i$ continuous I events in a cycle is: 


$$
E\left(N_{I}\right)=\sum_{i=1}^{\infty} \sum_{j=1}^{\infty} i P\left(N_{I}=i, N_{B U}=j\right)=\frac{1}{1-e^{-G a}}
$$

The possibility of $E\left(N_{B U}\right)$, the average number of $j$ continuous BU events in a cycle is:

$$
E\left(N_{B U}\right)=\sum_{i=1}^{\infty} \sum_{j=1}^{\infty} j P\left(N_{I}=i, N_{B U}=j\right)=\frac{1}{1-e^{-G(1+3 a)}}
$$

The average length of time slot that information packet has been successfully sent in a cycle is:

$$
\begin{aligned}
& E\left(N_{U}\right)=\frac{P(n=1)}{1-P(n=0)}=\frac{q_{a}^{1}}{1-q_{a}^{0}}=\frac{G a e^{-G a}}{1-e^{-G a}} \\
& E(U)=E\left(N_{U}\right) \times 1=\frac{P(n=1)}{1-P(n=0)}=\frac{G a e^{-G a}}{1-e^{-G a}}
\end{aligned}
$$

The average length of BU event is:

$$
E(B U)=E\left(N_{B U}\right) \times(1+3 a)=\frac{1+3 a}{e^{-G(1+3 a)}}
$$

In (6), $(1+3 a)$ represents the length of information packet whether it transmitted successfully or not in the TP cycle.

The average length of I event is:

$$
E(I)=E\left(N_{I}\right) \times a=\frac{a}{1-e^{-G a}}
$$

The throughput of the non-persist CSMA protocol with functions of monitoring is:

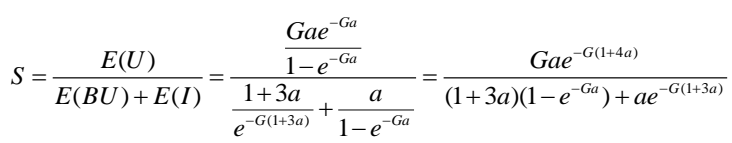

\section{Improved model analyses}

As is known, the throughput of traditional non-persist CSMA protocol is $S=\frac{G a e^{-G a}}{1-e^{-G a}+a}$ [3]. After introducing the algorithm of modified binary tree conflict resolution, in a cycle, for channel ${ }^{i}$, the length of time slot that information packet sent successfully occupies the effective information packet is:

$$
E\left(U_{i}^{*}\right)=E\left(N_{U_{i}}\right) \times(1+3 a)=\frac{a G_{i} e^{-a G_{i}}(1+3 a)}{1-e^{-a G_{i}}}
$$

In a cycle, for channel $i$, the average effective length that $x(x>=2)$ collision packets have been successfully divided and retransmitted is:

$$
E\left(N_{B x_{i}}\right)=\sum_{x=2}^{\infty} \frac{\left[G_{i}(1+3 a)\right]^{x} e^{-G_{i}(1+3 a)}}{x !\left(1-e^{-a G_{i}}\right)}
$$

$$
E\left(U_{B x_{i}}\right)=E\left(N_{B x_{i}}\right) \times x=\frac{G_{i}(1+3 a) e^{-G_{i}(1+3 a)}}{1-e^{-a G_{i}}}\left(e^{G_{i}(1+3 a)}-1\right)
$$

The process of the binary tree conflict resolution is:

If there are $x$ collision packets, they will choose the right or left time slot separately and randomly. Assuming its probability is $p_{i j}$. $p_{i j}^{\prime}$ is the probability that $i$ packets come to the left time slot and $j$ packets come to the right time slot. ${ }^{p_{i j}^{\prime}}$ is the probability that $j$ packets come to the left time slot and $i$ packets come to the right time slot [4].

$$
p_{i j}=p_{i j}^{\prime}+p_{j i}^{\prime}
$$

$$
p_{i(x-i)}=2 ! C_{x}^{i} C_{x-i}^{x-i} p^{x}=2 ! C_{x}^{i} p^{x} \quad, \quad i=0,1, \ldots,[N / 2]
$$

where ${ }^{x}$ is the odd number.

$$
p_{(x / 2)(x / 2)}=C_{x}^{x / 2} p^{x} \text {, where } x \text { is the even number. }
$$

The average length of successfully resolve $x$ collision packets is:

$$
\begin{gathered}
E\left(L_{x}\right)=p_{0 x}\left[1+E\left(L_{x}\right)\right]+p_{1(x-1)}^{\prime}\left[1+E\left(L_{x-1}\right)\right]+p_{(x-1) 1}^{\prime}\left[2+E\left(L_{x-1}\right)\right]+\sum_{i=2}^{[x / 2]} p_{i(x-i)}\left[1+E\left(L_{i}\right)+E\left(L_{x-i}\right)\right] \\
E\left(L_{x}\right)=\frac{p_{0 N}+p_{1(x-1)}^{\prime}\left[3+2 E\left(L_{x-1}\right)\right]+\sum_{i=2}^{[x / 2]} p_{i(x-i)}\left[1+E\left(L_{i}\right)+E\left(L_{x-i}\right)\right]}{1-p_{0 x}} \\
E\left(B x_{i}\right)=E\left(N_{B x}\right)(1+3 a) \times\left(x+E\left(L_{x}\right)\right)=\sum_{x=2}^{\infty} \frac{\left[G_{i}(1+3 a)\right]^{x} e^{-G_{i}(1+3 a)}(1+3 a)}{x !\left(1-e^{-a G_{i}}\right)} \bullet\left(x+E\left(L_{x}\right)\right)
\end{gathered}
$$

The throughput of the non-persist CSMA protocol based on binary tree conflict resolution with functions of monitoring of for channel $i$, is:

$$
S_{i}=\frac{E\left(U_{i}\right)+E\left(U_{B x_{i}}\right)}{E\left(U_{i}^{*}\right)+E\left(B x_{i}\right)+E\left(I_{i}\right)}
$$

As is known to all, $G_{1}=G_{2}=G_{3}=\ldots=G_{i}=\ldots=G_{N}, S=\sum_{i=1}^{N} \frac{E\left[U_{i}\right]}{E\left[U_{i}^{*}\right]+E\left[B_{i}\right]+E\left[I_{i}\right]}$ [5], then the system throughput of the discrete time non-persistent CSMA protocol with functions of monitoring and multichannel mechanism based on binary tree conflict resolution is $S=N S_{i}$.

The system throughput of the $l$ priority business is $S_{p l}=\left(\sum_{i=1}^{l} \frac{1}{N-i+1}\right) S_{i}$ 


\section{SIMULATION}

From the above analysis, the expression of the system throughput under the discrete time non-persistent CSMA protocol with functions of monitoring and multichannel mechanism based on binary tree conflict resolution and other parameters are got. With the simulation tool-MATLAB R2010a, system simulation and theoretical analysis are under the same working conditions. The simulation results are shown in Figure 2 to Figure 7. By default $a=0.01$.

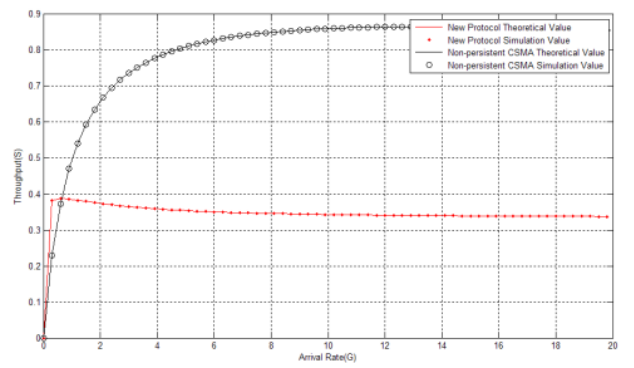

FIGURE II. THE COMPARISON BETWEEN THE NEW PROTOCOL AND THE TRADITIONAL PROTOCOL

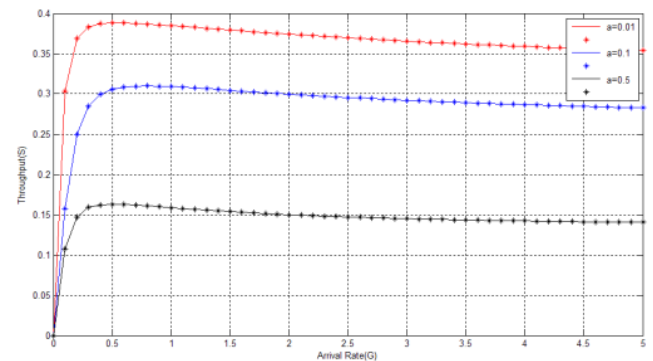

FIGURE III. WHEN $a=0.01, a=0.1, a=0.5$, THE NEW MODEL

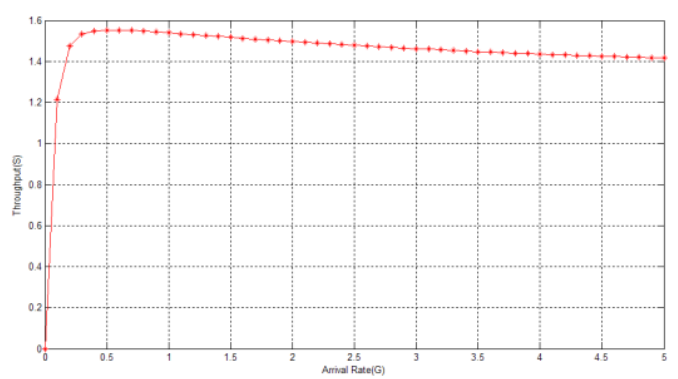

FIGURE IV. THE SYSTEM THROUGHPUT OF 4 CHANNELS

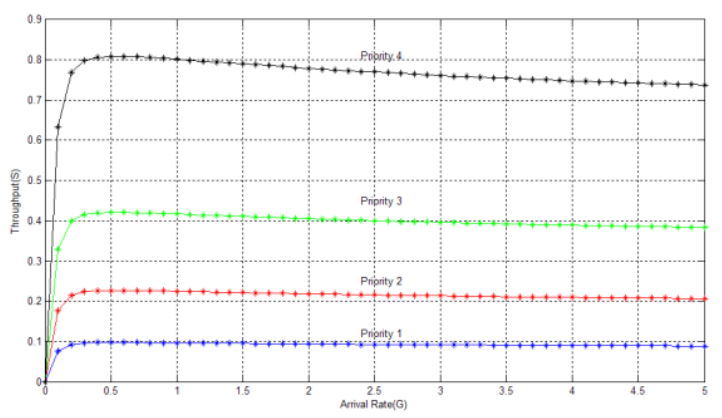

FIGURE V. THE COMPARISON OF 4 CHANNELS WITH DIFFERENT PRIORITIES

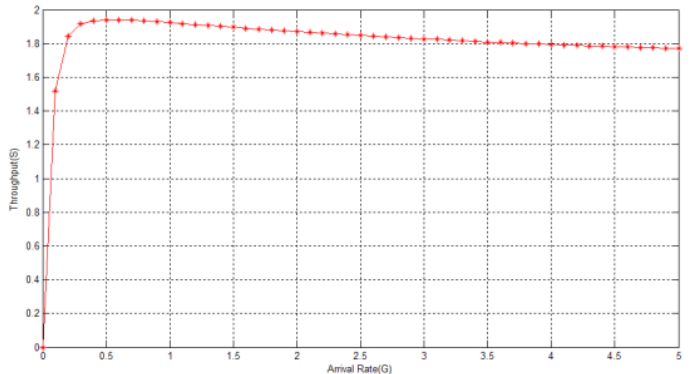

FIGURE VI. THE SYSTEM THROUGHPUT OF 5 CHANNELS

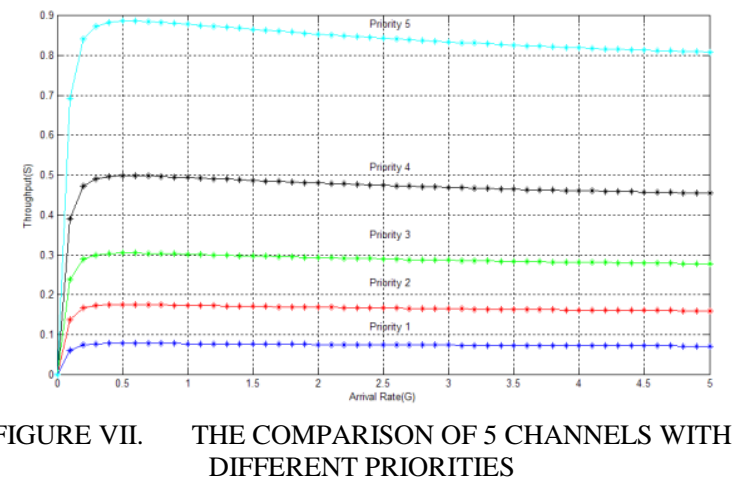

In Figure 2, the simulation values of system throughput under the new protocol and the traditional non-persistent CSMA protocol are consistent with the theoretical ones. Due to adding the monitoring information takes up more network resources, the system throughput of the new protocol is smaller than traditional non-persistent CSMA protocol. But when the arrival rate is less than 0.65 , the new protocol's system throughput is bigger than traditional non-persistent CSMA protocol. When the arrival rate is large enough, the system throughput of the new protocol is stable.

In Figure 3, when the arrival rate changes, the throughput of the new protocol is also changed; if the arrival rate become increases, the value of throughput will decrease.

Figure 4 through Figure 7, with the number of channels increases, the value of the new protocol's system throughput also increases; the priority is higher, the corresponding channel can get more network resources; network resources utilization has been greatly improved.

\section{CONCLUSIONS}

The new protocol, using the average cycle method, gets the precise mathematical expressions of system throughput and other related indicators through rigorous mathematical derivation. Through the simulation, the correctness of the theory is verified. By adding the monitoring information to improve the system controllability takes up more network resources, the throughput under the non-persistent CSMA with functions of monitoring is smaller than traditional nonpersistent CSMA protocol's. Modified binary tree conflict resolution algorithm is used to reduce the collision rate of the system; the use of multichannel and the priority mechanism improves the throughput of the new protocol. The utilization of network resources has been greatly improved and the system becomes more robust. 


\section{ACKNOWLEDGMENTS}

This work was supported by the National Natural Science Foundation of China (6146053, 61072079); Natural Science Foundation of Yunnan Province (2010CD023); The Financial Support of Yunnan University (No.XT412004).

\section{REFERENCES}

[1] G. P. Halkes,T. Dam,K. G. Langendoen. Comparing Energy-Saving MAC Protocols for Wireless Sensor Networks[J]. Mobile Networks and Applications . 2005 (5) :721-725.

[2] Yuhong Zhang, Wei Li. Modeling and energy consumption evaluation of a stochastic wireless sensor network[J]. EURASIP Journal on Wireless Communications and Networking, 2012(1): 79-82.

[3] Zhao Dongfeng. Study on A New Method for Continuous-time Systems of Random Access Channel [J]. Journal of Electronics, 1999, 21(1): 3741.

[4] Zhao Dongfeng, Li Bihai, Zheng Sumin. Study on a New Method for the Slotted Access Channel [J]. Journal of Electronics, 1997, 19(6):814-819.

[5] Yi Shang, Hongchi Shi. Flexible Energy Efficient Density Control on Wireless Sensor Networks[J]. International Journal of Distributed Sensor Networks, 2007, 3(1): 101-120. 